\title{
Potential relationship between Sirt3 and autophagy in ovarian cancer (Review)
}

\author{
YUCHUAN SHI $^{1 *}$, RUNHUA HE $^{1 *}, \mathrm{YU} \mathrm{YANG}^{2}, \mathrm{YU} \mathrm{HE}^{1}$, LEI ZHAN ${ }^{1,3}$ and BING WEI ${ }^{1}$ \\ ${ }^{1}$ Department of Gynaecology and Obstetrics, The Second Affiliated Hospital of Anhui Medical University; \\ ${ }^{2}$ Department of Cardiology, The First Affiliated Hospital of Anhui Medical University, Hefei, Anhui 230601; \\ ${ }^{3}$ Department of Obstetrics and Gynecology, Reproductive Medicine Center, \\ The First Affiliated Hospital of Anhui Medical University, Hefei, Anhui 230022, P.R. China
}

Received February 7, 2020; Accepted July 30, 2020

DOI: $10.3892 / \mathrm{ol} .2020 .12023$

\begin{abstract}
Sirtuin 3 (Sirt3) is an important member of the sirtuin protein family. It is a deacetylase that was previously reported to modulate the level of reactive oxygen species (ROS) production and limit the extent of oxidative damage in cellular components. As an important member of the class III type of histone deacetylases, Sirt3 has also been documented to mediate nuclear gene expression, metabolic control, neuroprotection, cell cycle and proliferation. In ovarian cancer (OC), Sirt3 has been reported to regulate cellular metabolism, apoptosis and autophagy. Sirt3 can regulate autophagy through a variety of different molecular signaling pathways, including the p62, 5'AMP-activated protein kinase and mitochondrial ROS-superoxide dismutase pathways. However, autophagy downstream of Sirt3 and its association with OC remains poorly understood. In the present review, the known characteristics of Sirt3 and autophagy were outlined, and their potential functional roles were discussed. Following a comprehensive analysis of the current literature, Sirt3 and autophagy may either serve positive or negative roles in the regulation of $\mathrm{OC}$. Therefore, it is important to identify the appropriate expression level of Sirt3 to control the activation of autophagy in OC cells. This strategy may prove to be a novel therapeutic method to reduce the mortality of patients with OC. Finally, potential research directions into the association between Sirt3 and other signaling pathways were provided.
\end{abstract}

Correspondence to: Dr Bing Wei or Dr Lei Zhan, Department of Gynaecology and Obstetrics, The Second Affiliated Hospital of Anhui Medical University, 678 Furong Road, Hefei, Anhui 230601, P.R. China

E-mail: 517275960@qq.com

E-mail: 499329901@qq.com

${ }^{*}$ Contributed equally

Key words: sirtuin 3, ovarian cancer, autophagy

\section{Contents}

1. Introduction

2. Sirt3 in OC

3. Autophagy in $\mathrm{OC}$

4. The relationship between Sirt3 and autophagy

5. Potential relationship between Sirt3 and autophagy in OC

6. Conclusion

\section{Introduction}

Ovarian cancer (OC) is a life-threatening malignancy that represents $3.6 \%$ female malignancies worldwide (1). It currently ranks as the seventh most common type of gynecological cancer and 20th as the most common type of cancer worldwide. OC has the highest mortality of all gynecologic malignancies $(1,2)$. According to a recent report, it was estimated that there were 295,414 new cases of OC diagnosed in 2018 and 184,799 cases of mortality resulting from this disease worldwide (3). Tumor debulking surgery followed by platinum and paclitaxel chemotherapy is currently the standard clinical treatment of OC (4). However, the survival rate of patients with advanced OC remains at $\sim 30 \%$, with the primary reasons being late discovery and chemoresistance. In particular, chemoresistance is mediated by both the tumor microenvironment and inherent resistance of OC cells to chemotherapy (5). Therefore, enhancement of responses to current treatment and the development of novel therapeutic strategies are urgently required to improve the survival rate.

Autophagy is a protective, catabolic process that operates to maintain intracellular homeostasis by recycling organelles and macromolecules (6). During this process, defective or aged organelles and other cytoplasmic components are enclosed by double-membrane vesicles to form autophagosomes. This then fuses with a lysosome where the vesicular contents are degraded into amino acids, lipids and carbohydrates by the lysosomal enzymes. The degradation products are in turn recycled to make new proteins and organelles (7). A basal level of autophagy is in operation under physiological conditions (8). However, downregulation or upregulation of autophagy induced by stress factors, including alterations in the levels of growth factors, hypoxia and cytotoxic damage, can result in cell death 
or cell adaptation in response (9). Autophagy also appears to serve contradictory roles in the development of cancer. Evidence exists reporting that inhibition of genes associated with autophagy can promote tumor development, whereas the expression of proteins associated with autophagy has also been demonstrated to result in inhibitory effects in several types of cancers (10-14). Therefore, autophagy can exert antitumor effects, but in contrast cancer cells may survive cellular stress in adverse microenvironments by utilizing autophagy, thereby promoting the development of tumors (15). In addition, autophagy is somewhat considered to be a double-edged sword in the clinical field of cancer. Promotion of autophagy can induce cell death, in a manner that is similar to apoptosis, whilst protective cellular autophagy has also been reported to be the major underlying cause of therapy resistance among cancer cells. Therefore, increasing the sensitivity of cancer cells to anticancer therapy by inhibiting autophagy remains a viable option (16).

Sirtuins are nicotinamide adenine dinucleotide $\left(\mathrm{NAD}^{+}\right)$-dependent histone deacetylases that are highly conserved among eukaryotic organisms, of which seven isoforms exist in mammals (17-19). They serve important roles in a number of biological and pathological processes. In particular, Sirt3 is localized to mitochondria, where it modulates the production of reactive oxygen species (ROS) to limit oxidative damage in cellular components (20). A wide range of important biological activities have been documented to be controlled by Sirt3, including the regulation of nuclear gene expression, metabolism, neuroprotection, cardiovascular disease, cancer cell cycle progression, and cell proliferation and apoptosis (21-24). Previously, the role of Sirt3 as a hallmark of cancer has been attracting significant research attention (25). Metabolism is an important caveat to cancer development (26), such that ATP is required for the maintenance of intracellular metabolic activity (27). A number of metabolic processes have been revealed to be regulated by Sirt3, including fatty-acid oxidation and oxidative phosphorylation $(28,29)$. These observations suggested that Sirt3 could be a key regulator of cancer physiology.

Currently, there are a number of studies on autophagy and Sirt3 in cardiovascular diseases, neuronal diseases and hepatotoxicity (30-32). However, almost no article has conducted research on the relationship between autophagy, OC and Sirt3. Therefore, the present review primarily discussed the potential relationship between Sirt3 and autophagy in OC, with the aim to provide a possible novel direction for $\mathrm{OC}$ research and therapeutic strategies.

\section{Sirt3 in OC}

OC poses a significant threat to the health of women worldwide, and is a disease in which Sirt3 has been reported serve a regulatory role. Of note, this disease is gradually becoming the leading cause of mortality associated with gynecological cancer worldwide in both developing and developed countries (1). Several reports have suggested that OC is regulated by Sirt3 using a multitude of mechanisms, which is summarized in this section.

In a previous study, it was found that muscle tissues after exercise exhibit elevated expression levels of the Sirt3 protein, which gave rise to the hypothesis that the expression of Sirt3 is regulated by energy metabolism (33). Energy metabolism is also associated with the regulation of tumor growth and metastasis. Sirt3 is regarded as a tumor suppressor, due to a previous finding that its expression is reduced in tumors $(34,35)$. The activation of cellular autophagy and apoptosis was demonstrated to be controlled by Sirt3 via the regulation of several signaling pathways during the development of OC. A previous study found that expression of the Sirt3 protein was significantly downregulated in OC tissues and in highly metastatic HO-8910PM cell lines $(35,36)$. In addition, Xiang et al (37) demonstrated that the activation of Sirt3 exerted a proapoptotic function in SKOV3 cells. These findings suggested that overexpression of Sirt 3 can induce OC cell death. In terms of mitochondrial dynamics, a previous study revealed that stabilization of Sirt 3 can increase mitochondrial biogenesis and cristae remodeling in OC tissues (38). Additionally, stabilization of optic atrophy protein 1 , which increased resistance to apoptosis, was demonstrated to be regulated by increasing the expression of Sirt3 and prohibitin 2 (38). Activation of Sirt3 has also been found to enhance the sensitivity of OC cells to cisplatin (39), rendering Sirt3 to be a novel therapeutic target. In addition, Sirt3 was reported to be a favorable independent prognostic factor for overall survival for patients with serious OC in a previous study (40). In conclusion, Sirt3 serves an important role in the development of OC, with therapeutic and prognostic implications.

\section{Autophagy in OC}

Autophagy is a catabolic process that serves to maintain intracellular homeostasis by recycling damaged cellular organelles (41), which has been studied since the 1960s (42). Over the past decades, the molecular mechanisms underlying this process have been revealed gradually. It has been suggested that autophagy is a common phenomenon that occurs during both physiological and pathological conditions. According to the sizes of the substrates involved and degradation rate, autophagy can be divided into three sub-categories: i) Macro-autophagy; ii) microautophagy; and iii) chaperone-mediated autophagy (43). Although different types of autophagy utilize distinct mechanisms to degrade lysosomal proteins, common underlying characteristics remain (44). The autophagy pathway consists of the following six steps: i) Initiation of autophagy; ii) biogenesis of the phagophore; iii) expansion of the phagophore; iv) formation of the autophagosome; v) fusion with the lysosome; and vi) reformation of the lysosome (45). Autophagy is constitutively active at low levels in all cell types under physiological conditions, but can be potentiated by nutrient deprivation, hypoxia, endoplasmic reticulum stress, pathogenic toxicity and immune injury, to maintain intracellular homeostasis (46). Previous studies have demonstrated that autophagy serves an important role in the pathological processes of various diseases, including neurodegenerative and cardiovascular disease, cancer, infectious diseases and immune deficiency (47-50). Autophagy has been described as a double-edged sword, since it can exert both tumor suppression and growth promotion (51). In this section, the mechanism of autophagy in $\mathrm{OC}$ is discussed in detail. 
OC ranks as the most lethal gynecological malignancy, with high morbidity and mortality. Autophagy serves an important role in OC through the expression of autophagy-associated proteins, including beclin-1, microtubule-associated proteins 1A/1B light chain 3B (LC3) and p53. Beclin-1 is a tumor suppressor, which is an important checkpoint protein that is involved in autophagy and tumor cell apoptosis $(52,53)$. It has been reported to mediate various functions in tumors, where its expression level varies depending on the type of malignancy. A previous study revealed that the expression level of beclin-1 was higher in ovarian epithelial cancers, which can be used as an independent risk factor for the prognosis of patients with this disease (54). In addition, other proteins linked to autophagy can mediate functions in OC, which are in turn associated with a number of signaling pathways, including the PI3K/AKT/mTOR and p53 signaling pathway. These are summarized in this section.

Autophagy-related PI3K/AKT/mTOR signaling pathway. The autophagic process in OC is regulated by a number of factors. The PI3K/AKT/mTOR pathway has frequently been associated with the majority of human malignancies, studies have demonstrated that other signaling pathways related to oncogenesis are also caused by dysregulations in this signaling pathway (55-57). The reason for this dysregulation is manifold, including mutations in PI3K, AKT overexpression and the sustained activation of tyrosine kinase growth factor receptors (58). The PI3K/AKT/mTOR signaling pathway has been documented to regulate cell survival, proliferation, growth, transcription, angiogenesis and metabolism (55-57). In 70\% cases of OC, the PI3K/AKT/mTOR pathway has been revealed to be constitutively activated, which has been considered to be a therapeutic target (59). To verify if the PI3K/AKT pathway is involved in OC cell proliferation, Hu et al (60) treated OC cell lines with the specific PI3K inhibitor LY294002 and established a mouse model of OC. Proliferation of OC cells can be significantly inhibited by LY294002 treatment in vitro (61). In addition, other studies have found that AKT inhibitors can prevent the function of mTORC1/2 and AKT itself to inhibit the PI3K signaling cascade (61-63). In another study, Ichikawa et al (64) found that the cytotoxic effects of chemotherapeutic agents can be effectively enhanced by co-treatment with the selective non-competitive AKT inhibitor TAS-117 in vivo OC models. In OC, the PI3K/AKT/mTOR signaling pathway is frequently activated, which indicates that the inhibition of this signaling pathway could prove to be a potential avenue of treatment strategies, either as a monotherapy or in combination with other chemotherapeutic agents.

p53 signaling pathway. $\mathrm{p} 53$ is a key tumor suppressor that serves an important regulatory role in autophagy in mammalian cells (65). Expression of the p53 gene is activated by various intracellular events, including DNA damage, hypoxia and oncogene activation, to prevent cell damage and maintain cellular integrity. Numerous types of modifications, including acetylation, methylation, phosphorylation and ubiquitination, are involved in regulating the activation of p53 on a molecular level $(66,67)$. p53 target genes negatively regulate mTOR activity, which in turn induces autophagy in the nucleus. p53 can promote autophagy by inhibiting
mTOR via the 5'AMP-activated protein kinase (AMPK) pathway (68). In addition, p53 can also induce autophagy by activating damage-regulated autophagy modulator (69). Several studies have revealed that autophagy may be triggered by the inactivation of cytoplasmic p53, such that extranuclear p53 is an effective inhibitor of autophagy $(70,71)$. A clinical study previously demonstrated the upregulated expression of p53 in OC, where it was revealed that at later tumor stages, the rate of p53-positive expression was higher (72). These results suggested that p53 serves an important role in the development of OC. Additionally, another study previously found that silencing the p53 signaling pathway may suppress proliferation whilst facilitating apoptosis and cisplatin chemosensitivity in OC cells (73). Therefore, these aforementioned findings suggested that the efficacy of chemotherapeutic treatments for OC can be improved by inhibiting the p53-induced autophagic process.

\section{Relationship between Sirt3 and autophagy}

Sirt3 belongs to the $\mathrm{NAD}^{+}$-dependent protein deacetylase family and is responsible for the majority of mitochondrial protein deacetylation (74). It has been reported to serve an important role in almost every aspect of mitochondrial biology, such as mitochondrial biogenesis and mitochondrial oxidative stress (30,75-77). Although Sirt3 is located in mitochondria, almost every major key component of autophagy is cytosolic. Therefore, a type of communication mechanism must exist between mitochondria and cytosol during the regulation of Sirt3 activity and autophagy. In previous years, accumulating evidence has suggested that there are a series of signaling pathways between Sirt3 and autophagy, including the p62, PI3K/AKT, AMPK-mTOR pathway and the mitochondrial ROS-superoxide dismutase 2 (SOD2) pathway. This section aimed to summarize the known information on its mechanism (Fig. 1).

Sirt3 and p62.p62 is the selective cargo receptor for autophagy that is indispensable for the degeneration of misfolded proteins. Reductions in p62 expression have been previously reported to activate autophagy (78). In addition, p62 is considered to be a marker of autophagic flux due to its differential expression profiles in association with other proteins linked to autophagy (79). As for Sirt3, a number of studies have indicated that there is a close relationship between Sirt3 and the p62-autophagy pathway. A previous study demonstrated that treatment with ANXA1sp, an annexin-A1 bioactive peptide, reduced the expression of p62 and concomitantly upregulated the expression of the mitochondrial protein deacetylase Sirt3 (80). This suggested that Sirt3 is involved in autophagy by downregulating p62. Tong et al (81) also reported a similar finding, where liraglutide treatment downregulated the expression of p62 to promote autophagy via the Sirt1/Sirt3-forkhead box O3-p62 pathway in mice that were fed a normal-fat diet or high-fat diet. Additionally, a previous investigation focusing on the effects of melatonin on diabetic cardiomyopathy revealed that melatonin can upregulate autophagy by increasing the expression of LC3-II whilst downregulating that of p62 (82), with the macrophage stimulating $1 /$ Sirt 3 signaling pathway being the likely associated mechanism. These aforementioned 


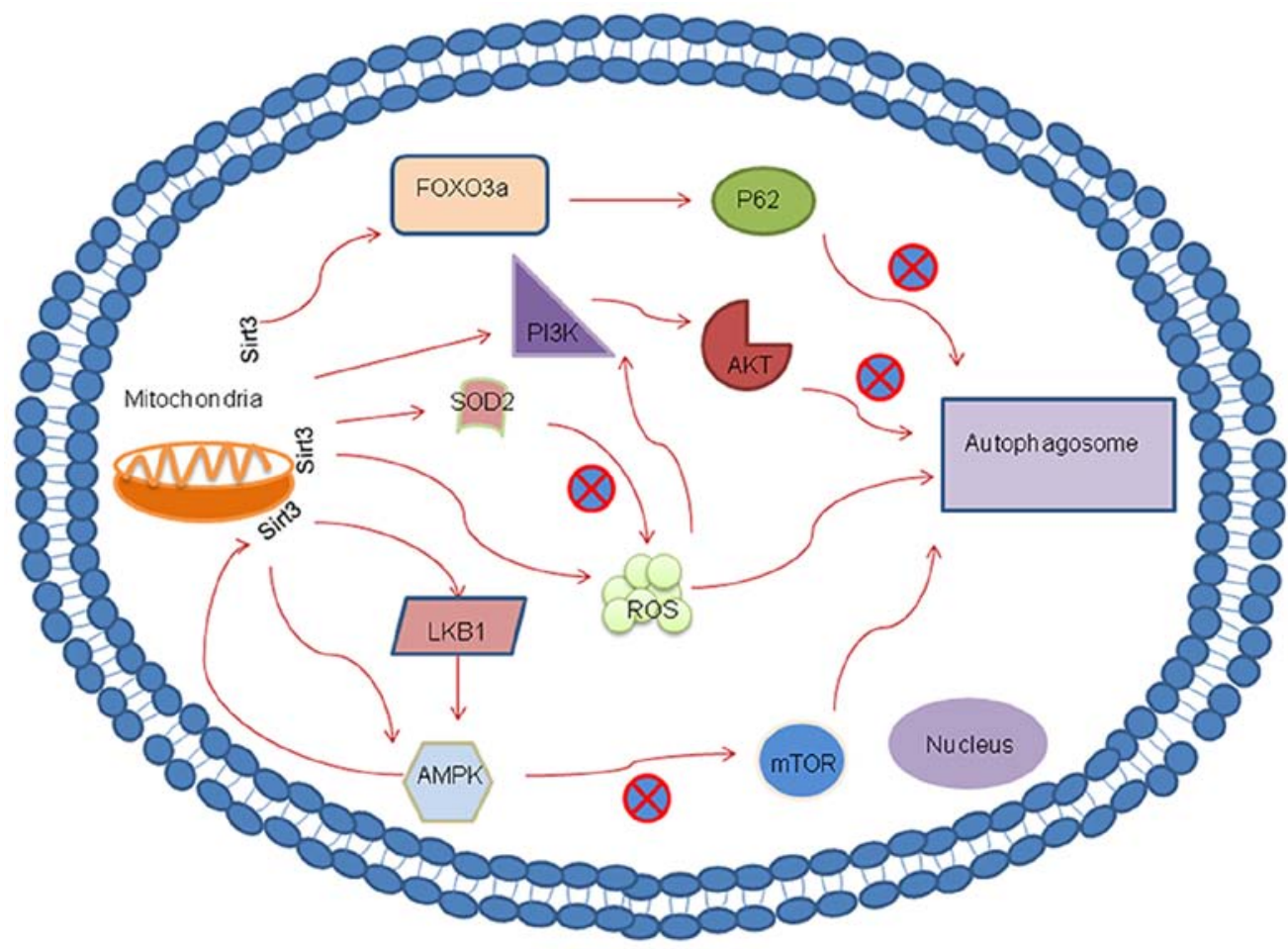

Figure 1. Potential signaling pathways underlying Sirt3-mediated regulation of autophagy. Sirt3 is involved in autophagy via the downregulation of p62. The Sirt3/FOXO3a/p62 and macrophage stimulating 1/Sirt3 signaling pathways can downregulate p62 expression. In addition, Sirt3 can directly activate PI3K/AKT signaling to inhibit autophagy. Mechanistically, Sirt3 indirectly controls the hyperactivation of AKT by regulating mitochondrial ROS production and ROS-mediated Ras-PI3K-AKT activation. Sirt3 can activate LKB1, such that the LKB1-AMPK-mTOR pathway serves an important role in autophagy. AMPK can inhibit mTOR directly to promote autophagy whilst also acting upstream of Sirt3. ROS can act as a trigger signal to induce autophagy through autophagy related protease 4. Autophagy may be suppressed by Sirt3 through the regulation of mitochondrial ROS production. The Sirt3/SOD2 pathway is involved in mitochondrial-derived $\mathrm{O}_{2}$-stimulated autophagic cell death. Sirt3-mediated deacetylation can significantly increase the activity of SOD2 to ultimately reduce intracellular ROS levels. Sirt3, sirtuin; FOXO3a, forkhead box O3; ROS, reactive oxygen species; LKB1, liver kinase B1; AMPK, 5'AMP-activated protein kinase; SOD2, superoxide dismutase 2.

pharmacological studies suggested that Sirt3 is a potent activator of autophagy. Supporting this, Xiang et al (83) found that small interfering (si)RNA-mediated silencing of Sirt3 gene expression inhibited the process of autophagy and p62 degradation in human umbilical vein endothelial cells.

Sirt3 and PI3K/AKT. The PI3K/AKT pathway serves an important inhibitory role in autophagy, and is also involved in a variety of physiological and pathological processes $(84,85)$. By regulating the PI3K/AKT pathway, Sirt3 can function as an autophagy suppressor. A previous report that investigated hepatocellular carcinoma found that the expression levels of Sirt3 was higher in adjacent non-cancerous tissues compared with those in hepatocellular carcinoma tissues (86). Furthermore, using Sirt3 knockdown, this previous study also demonstrated that Sirt3 may serve as a suppressor of autophagy in hepatocellular carcinoma by targeting the PI3K/AKT pathway. Another study revealed a consistent finding, where Sirt3 functioned as a growth suppressor in prostate cancer by inhibiting the activation of PI3K/AKT both in vitro and in vivo (87). This previous study also showed that the progression of prostate cancer may be downregulated via the Sirt3/AKT/c-Myc signaling axis (87). Wang et al (88) demonstrated that glioblastoma multiforme cell growth was inhibited through the Sirt3/p53-mediated PI3K/AKT/ERK and mitochondrial signaling pathway. In addition, Sirt3 was reported to indirectly regulate AKT hyperactivation by regulating mitochondrial ROS production upstream of the ROS-mediated Ras-PI3K-AKT activation (89). In summary, Sirt 3 can regulate autophagy by either directly or indirectly regulating the PI3K/AKT pathway.

Sirt 3 and AMPK. AMPK is a conserved a intracellular energy sensor that mediates energy homeostasis by regulating lipid and glucose metabolism (90). Dysregulation of AMPK has previously been associated with accelerated aging (91). In previous years, a number of studies have attempted to unravel the mechanism underlying the regulation of autophagy. There is increasing consensus that AMPK and mTOR are regarded as the main regulators of autophagic degradation $(92,93)$. A close mechanistic relationship has been reported to exist between Sirt3 and the AMPK-mTOR-autophagy pathway. Zhao et al (94) previously found that induction of autophagy was directly controlled by Sirt3 via the AMPK-mTOR pathway during acute kidney injury. In addition, another study reported that Sirt3-indcued autophagy protected against oxygen and glucose deprivation by regulating the AMPK-mTOR pathway (30).

Previous studies have found that AMPK can negatively regulate mTORC1 activity via two different mechanisms. AMPK can phosphorylate Thr1227 and Ser1345 residues to activate tuberous sclerosis complex (TSC) 2, thereby promoting the formation of the TSC1/TSC2 heterodimer to inhibit mTORC1 activity (95). By contrast, AMPK can also phosphorylate regulatory-associated protein of mTOR on its Ser722 and Ser792 


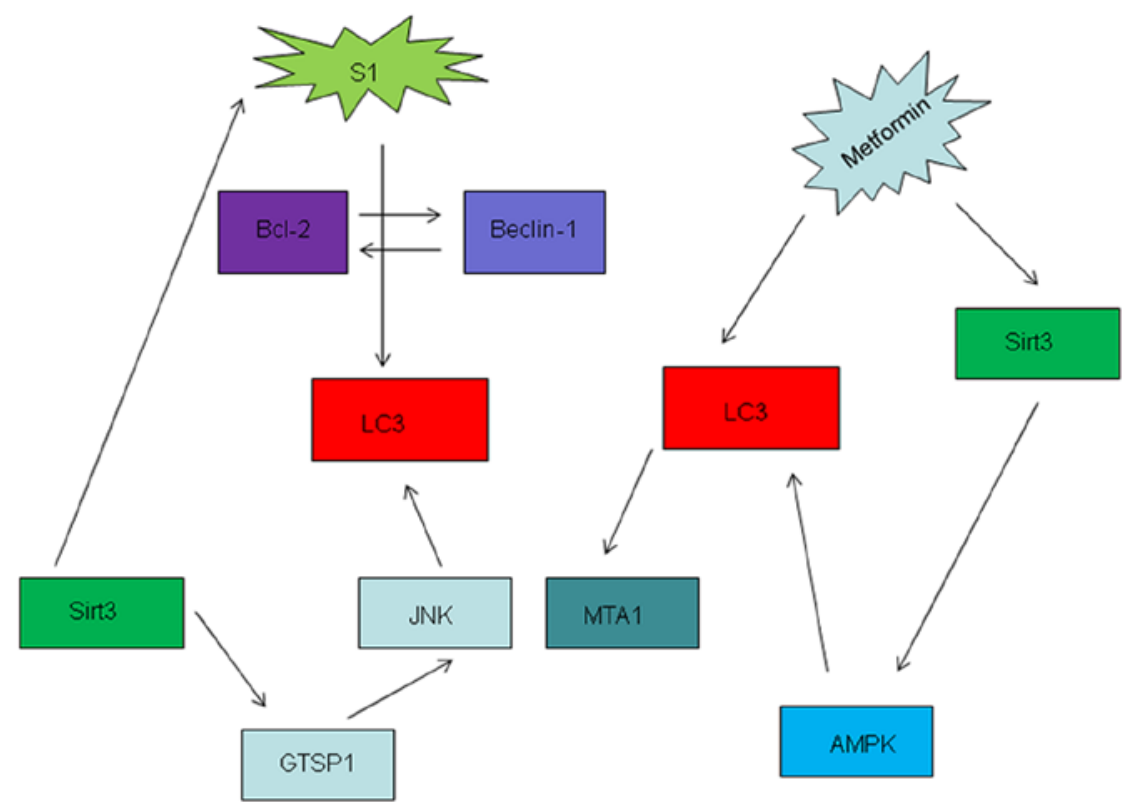

Figure 2. Relationship between Sirt3 and autophagy in ovarian cancer. Metformin-induced overexpression of Sirt3 activates AMPK, which in turn increases the expression of LC3. This results in the elevation in the expression of proteins associated with cell migration, such as MTA1. S1 is a novel pan Bcl-2 inhibitor that has been documented to activate autophagy by interrupting the interaction between Bcl-2 and Beclin1. Sirt3 is involved in the regulation of autophagy via the GTSP1/JNK/autophagy pathway. S1 can promote the expression of JNK3. Knocking down Sirt3 expression can alleviate S1-induced apoptosis. MTA1, metastasis-associated protein; GTSP, glutathione S-transferase P; LC3, microtubule-associated proteins 1A/1B light chain 3B; Sirt3, sirtuin; AMPK, 5'AMP-activated protein kinase.

residues to inhibit mTORC1 (96). Liver kinase B1 (LKB1) is a tumor suppressor and an upstream regulator of AMPK that has an essential role in the control of redox homeostasis (97). Incidentally, LKB1 can also be activated by Sirt3. A previous investigation into a rotenone-induced SH-SY5Y cell injury model found that the overexpression of Sirt3 promoted LKB1 phosphorylation, which activated AMPK and reduced the phosphorylation of mTOR. This observation suggested that the LKB1-AMPK-mTOR pathway can be regulated by Sirt3 (98). In addition, another previous study documented that activation of the LKB1-AMPK-mTOR-mediated autophagy signaling pathway can be induced by Sirt3 (99). Notably, AMPK can function upstream of Sirt3 during the regulation of insulin sensitivity in skeletal muscle via the AMPK-peroxisome proliferator-activated receptor $\gamma$ coactivator $1 \alpha$-Sirt3 autophagy signaling pathway in Sirt3-/-mice (100). Collectively, this indicated that Sirt3 and autophagy have a complex mutual regulatory relationship.

Sirt3 and ROS. ROS consists of a group of highly reactive chemical entities, including oxygen radicals, hydroxyl, peroxyl, alkoxyl, non-radicals, singlet oxygen and hydrogen peroxide. These molecules are primarily produced from redox transactions as part of the oxidative phosphorylation system in mitochondria (100). A number of studies have demonstrated that ROS can act as a signal to trigger autophagy through autophagy-regulating protease 4 , which serves as part of the autophagy process $(101,102)$. Autophagy may be suppressed by Sirt 3 via the regulation of mitochondrial ROS (mROS) production. Recently, a study investigating the potential antineoplastic properties of metformin combined with nelfinavir revealed that this drug combination can increase the expression level of Sirt3. This increment in Sirt3-mediated mROS production served a vital role in the autophagic mechanism within human cervical cancer cells (103). Upstream of ROS, SOD2 activity can moderately reduce cellular ROS levels. Sirt3-mediated deacetylation can significantly potentiate SOD2 activity to ultimately break down intracellular ROS (104). A previous study demonstrated that Sirt3 protein expression and activity was downregulated by cadmium $(\mathrm{Cd})$, which can also concurrently promote the acetylation of SOD2 to suppress its activity, thereby increasing mROS production (31). In summary, mitochondrial-derived ROS-dependent autophagic cell death can be induced by $\mathrm{Cd}$. Consistent with this notion, $\mathrm{Cd}$-induced hepatotoxicity has been reported to be alleviated by the protective properties of melatonin (31). In this particular study, the activity, but not the expression of Sirt3, was revealed to be enhanced by melatonin treatment (31). Additionally, melatonin was also demonstrated to inhibit mitochondria-derived $\mathrm{O}_{2}$ production, reduced the acetylation of SOD2 and suppressed autophagy (31). This finding suggested that melatonin exerts hepatoprotective effects by regulating mitochondria-derived $\mathrm{O}_{2}$-stimulated autophagic cell death via the Sirt3-SOD2 pathway. According to the aforementioned findings, SOD2 activity and intracellular mROS homeostasis may underlie the Sirt3 downregulation of autophagy (31).

\section{Potential relationship between Sirt3 and autophagy in OC}

Autophagy is a fundamental catabolic process that has been reported to be involved in the progression of a variety of diseases. It can serve a protective role in OC cells from cell death since it may enhance resistance to cisplatin (105). A previous investigation revealed that cisplatin treatment activated autophagy, whereas Bcl-2-associated athanogene 3 attenuated cisplatin resistance by inhibiting autophagy (106). 
However, recent studies have also suggested that autophagy can inhibit the growth of OC, since it has been found to promote OVCAR-3 cell death (107). By contrast, findings from another previous study suggested that inhibition of autophagy promoted the proliferation and invasion of $\mathrm{OC}$ cells via the PI3K/AKT/mTOR pathway (108). These findings demonstrated that autophagy is a double-edged sword in the regulation of OC physiology. Sirt3 serves an important role in the maintenance of intracellular homeostasis in OC. Previous studies have indicated that there is a close mutual regulatory relationship between Sirt3 and autophagy, which are linked by the aforementioned signaling pathways in the present review. Metformin-induced overexpression of Sirt3 promoted apoptosis and mitochondrial dysfunction whilst increasing the activation of AMPK in OC cell lines (109). In addition, metformin has been documented to promote autophagy in OC (110). S1, a novel Bcl-2 inhibitor, has also been reported to exert autophagic effects in $\mathrm{OC}$ by interrupting the interaction between $\mathrm{Bcl}-2$ and beclin1 in $\mathrm{OC}$ to promote apoptosis (111); however, high doses and longer exposure of S1 can overpower the protective function of autophagy and induce apoptosis $(112,113)$. Yang et al (114) previously found that S1 promoted JNK3 expression, thus increasing cell sensitivity to apoptosis. Sirt3 can also regulate autophagy via the glutathione S-transferase P/JNK/autophagy pathway, such that Sirt3 knockdown has been demonstrated to alleviate S1-induced apoptosis (Fig. 2) (37).

\section{Conclusion}

Autophagy serves an important role in recycling damaged organelles and maintaining intracellular homeostasis. Sirt3 is a potential therapeutic target, since it has been previously reported to activate autophagy. In the present review, although the potential relationship between Sirt 3 and autophagy in OC was explored, there remains an insufficient number of studies on this topic. The associated underlying mechanism in the Sirt3-induced autophagic process in OC remains unclear. With further study, novel insights into the molecular relationship between Sirt3 and autophagy may contribute to the development of novel therapeutic interventions for OC.

\section{Acknowledgements}

Not applicable.

\section{Funding}

The present project was mainly supported by the National Natural Science Foundation of China (grant no. 81802586).

\section{Availability of data and materials}

Not applicable.

\section{Authors' contributions}

YS and RH contributed to the conception of this manuscript and wrote the draft. YY contributed to the literature collection and preparation. $\mathrm{YH}$ contributed to the revision of this manuscript. LZ and BW provided funding and proofed the manuscript. All authors read and approved the final version of the manuscript.

\section{Ethics approval and consent to participate}

Not applicable.

\section{Patient consent for publication}

Not applicable.

\section{Competing interests}

The authors declare that they have no competing interests.

\section{References}

1. Torre LA, Trabert B, DeSantis CE, Miller KD, Samimi G, Runowicz CD, Gaudet MM, Jemal A and Siegel RL: Ovarian cancer statistics, 2018. CA Cancer J Clin 68: 284-296, 2018.

2. Hackshaw A, Gershenson D and Ledermann J: Mucinous ovarian carcinoma. N Engl J Med 381: e3, 2019.

3. Bray F, Ferlay J, Soerjomataram I, Siegel RL, Torre LA and Jemal A: Global cancer statistics 2018: GLOBOCAN estimates of incidence and mortality worldwide for 36 cancers in 185 countries. CA Cancer J Clin 68: 394-424, 2018.

4. Wang L, Wang Q, Xu Y and Han L: Advances in the treatment of ovarian cancer using PARP inhibitors and the underlying mechanism of resistance. Curr Drug Targets 21: 167-178, 2020.

5. Hansen JM, Coleman RL and Sood AK: Targeting the tumour microenvironment in ovarian cancer. Eur J Cancer 56: 131-143, 2016.

6. Liu J, Ren Y, Hou Y, Zhang C, Wang B, Li X, Sun R and Liu J: Dihydroartemisinin induces endothelial cell autophagy through suppression of the Akt/mTOR pathway. J Cancer 10: 6057-6064, 2019.

7. Ravikumar B, Sarkar S, Davies JE, Futter M, Garcia-Arencibia M, Green-Thompson ZW, Jimenez-Sanchez M, Korolchuk VI, Lichtenberg M, Luo S, et al: Regulation of mammalian autophagy in physiology and pathophysiology. Physiol Rev 90: 1383-1435, 2010.

8. Melendez A and Neufeld TP: The cell biology of autophagy in metazoans: A developing story. Development 135: 2347-2360, 2008.

9. Boya P, González-Polo RA, Casares N, Perfettini JL, Dessen P, Larochette N, Métivier D, Meley D, Souquere S, Yoshimori $\mathrm{T}$, et al: Inhibition of macroautophagy triggers apoptosis. Mol Cell Biol 25: 1025-1040, 2005.

10. Yue Z, Jin S, Yang C, Levine AJ and Heintz N: Beclin 1, an autophagy gene essential for early embryonic development, is a haploinsufficient tumor suppressor. Proc Natl Acad Sci USA 100: 15077-15082, 2003.

11. Coppola D, Khalil F, Eschrich SA, Boulware D, Yeatman T and Wang HG: Down-regulation of Bax-interacting factor-1 in colorectal adenocarcinoma. Cancer 113: 2665-2670, 2008.

12. Boteon YL, Laing R, Mergental H, Reynolds GM, Mirza DF, Afford SC and Bhogal RH: Mechanisms of autophagy activation in endothelial cell and their targeting during normothermic machine liver perfusion. World J Gastroenterol 23: 8443-8451, 2017.

13. Nussenzweig SC, Verma S and Finkel T: The role of autophagy in vascular biology. Circ Res 116: 480-488, 2015.

14. Liang C, Feng P, Ku B, Dotan I, Canaani D, Oh BH and Jung JU: Autophagic and tumour suppressor activity of a novel Beclin1-binding protein UVRAG. Nat Cell Biol 8: 688-699, 2006.

15. Bishop E and Bradshaw TD: Autophagy modulation: A prudent approach in cancer treatment? Cancer Chemother Pharmacol 82: 913-922, 2018.

16. Li YJ, Lei YH, Yao N, Wang CR, Hu N, Ye WC, Zhang DM and Chen ZS: Autophagy and multidrug resistance in cancer. Chin J Cancer 36: 52, 2017. 
17. Haigis MC, Deng CX, Finley LW, Kim HS and Gius D: SIRT3 is a mitochondrial tumor suppressor: A scientific tale that connects aberrant cellular ROS, the Warburg effect, and carcinogenesis. Cancer Res 72: 2468-2472, 2012.

18. Sack MN and Finkel T: Mitochondrial metabolism, sirtuins, and aging. Cold Spring Harb Perspect Biol 4: a013102, 2012.

19. Michan S and Sinclair D: Sirtuins in mammals: Insights into their biological function. Biochem J 404: 1-13, 2007.

20. Verdin E, Hirschey MD, Finley LW and Haigis MC: Sirtuin regulation of mitochondria: Energy production, apoptosis, and signaling. Trends Biochem Sci 35: 669-675, 2010.

21. Anderson KA and Hirschey MD: Mitochondrial protein acetylation regulates metabolism. Essays Biochem 52: 23-35, 2012.

22. Alhazzazi TY, Kamarajan P, Joo N, Huang JY, Verdin E, D'Silva NJ and Kapila YL: Sirtuin-3 (SIRT3), a novel potential therapeutic target for oral cancer. Cancer 117: 1670-1678, 2011.

23. Shi T, Wang F, Stieren E and Tong Q: SIRT3, a mitochondrial sirtuin deacetylase, regulates mitochondrial function and thermogenesis in brown adipocytes J Biol Chemy 280: 13560-13567, 2005.

24. Kong X, Wang R, Xue Y, Liu X, Zhang H, Chen Y, Fang F and Chang Y: Sirtuin 3, a new target of PGC-1alpha, plays an important role in the suppression of ROS and mitochondrial biogenesis. PLoS One 5: e11707, 2010.

25. Guarente L: Introduction: Sirtuins in aging and diseases. Methods Mol Biol 1077: 3-10, 2013.

26. Resendis-Antonio O, Checa A and Encarnacion S: Modeling core metabolism in cancer cells: Surveying the topology underlying the Warburg effect. PLoS One 5: e12383, 2010.

27. Madhok BM, Yeluri S, Perry SL, Hughes TA and Jayne DG: Targeting glucose metabolism: An emerging concept for anticancer therapy. Am J Clin Oncol 34: 628-635, 2011.

28. Hirschey MD, Shimazu T, Goetzman E, Jing E, Schwer B Lombard DB, Grueter CA, Harris C, Biddinger S and Ilkayeva OR: SIRT3 regulates mitochondrial fatty-acid oxidation by reversible enzyme deacetylation. Nature 464: 121-125, 2010.

29. Bagul PK, Katare PB, Bugga P, Dinda AK and Banerjee SK: SIRT-3 modulation by resveratrol improves mitochondrial oxidative phosphorylation in diabetic heart through deacetylation of TFAM. Cells 7: 235, 2018.

30. Dai SH, Chen T, Li X, Yue KY, Luo P, Yang LK, Zhu J, Wang YH, Fei Z and Jiang XF: Sirt3 confers protection against neuronal ischemia by inducing autophagy: Involvement of the AMPK-mTOR pathway. Free Radic Biol Med 108: 345-353, 2017

31. Pi H, Xu S, Reiter RJ, Guo P, Zhang L, Li Y, Li M, Cao Z, Tian L, Xie J, et al: SIRT3-SOD2-mROS-dependent autophagy in cadmium-induced hepatotoxicity and salvage by melatonin. Autophagy 11: 1037-1051, 2015.

32. Li Y, Ma Y, Song L, Yu L, Zhang L, Zhang Y, Xing Y, Yin Y and Ma H: SIRT3 deficiency exacerbates p53/Parkin-mediated mitophagy inhibition and promotes mitochondrial dysfunction: Implication for aged hearts. Int J Mol Med 41: 3517-3526, 2018.

33. Edgett BA, Hughes MC, Matusiak JB, Perry CG, Simpson CA and Gurd BJ: SIRT3 gene expression but not SIRT3 subcellular localization is altered in response to fasting and exercise in human skeletal muscle. Exp Physiol 101: 1101-1113, 2016.

34. Chen Y, Fu LL, Wen X, Wang XY, Liu J, Cheng Y and Huang J: Sirtuin-3 (SIRT3), a therapeutic target with oncogenic and tumor-suppressive function in cancer. Cell Death Dis 5: e1047, 2014

35. Dong XC, Jing LM, Wang WX and Gao YX: Down-regulation of SIRT3 promotes ovarian carcinoma metastasis. Biochem Biophys Res Commun 475: 245-250, 2016.

36. Yang Y, Cao Y, Chen L, Liu F, Qi Z, Cheng X and Wang Z: Cryptotanshinone suppresses cell proliferation and glucose metabolism via STAT3/SIRT3 signaling pathway in ovarian cancer cells. Cancer Med 7: 4610-4618, 2018.

37. Xiang XY, Kang JS, Yang XC, Su J, Wu Y, Yan XY, Xue YN, $\mathrm{Xu}$ Y, Liu YH, Yu CY, et al: SIRT3 participates in glucose metabolism interruption and apoptosis induced by BH3 mimetic S1 in ovarian cancer cells. Int J Oncol 49: 773-784, 2016.

38. Signorile A, De Rasmo D, Cormio A, Musicco C, Rossi R, Fortarezza F, Palese LL, Loizzi V, Resta L, Scillitani G, et al: Human ovarian cancer tissue exhibits increase of mitochondrial biogenesis and cristae remodeling. Cancers (Basel) 11: 1350, 2019.

39. Hou L, Wang R, Wei H, Li S, Liu L, Lu X, Yu H and Liu Z: ABT737 enhances ovarian cancer cells sensitivity to cisplatin through regulation of mitochondrial fission via Sirt3 activation. Life Sci 232: 116561, 2019.
40. Li J, Yue H, Yu H, Lu X and Xue X: Development and validation of SIRT3-related nomogram predictive of overall survival in patients with serous ovarian cancer. J Ovarian Res 12: 47, 2019.

41. Yang Y and Klionsky DJ: Autophagy and disease: Unanswered questions. Cell Death Differ 27: 858-871, 2020

42. Wilde L, Tanson K, Curry J and Martinez-Outschoorn U: Autophagy in cancer: A complex relationship. Biochem J 475 1939-1954, 2018

43. Glick D, Barth S and Macleod KF: Autophagy: Cellular and molecular mechanisms. J Pathol 221: 3-12, 2010.

44. Bednarczyk M, Zmarzly N, Grabarek B, Mazurek U and Muc-Wierzgon M: Genes involved in the regulation of different types of autophagy and their participation in cancer pathogenesis. Oncotarget 9: 34413-34428, 2018.

45. Zhi X, Feng W, Rong Y and Liu R: Anatomy of autophagy: From the beginning to the end. Cell Mol Life SciS 75: 815-831, 2018.

46. Burman C and Ktistakis NT: Autophagosome formation in mammalian cells. Semin Immunopathol 32: 397-413, 2010.

47. Schaeffer V, Lavenir I, Ozcelik S, Tolnay M, Winkler DT and Goedert M: Stimulation of autophagy reduces neurodegeneration in a mouse model of human tauopathy. Brain 135: 2169-2177, 2012.

48. Liao X, Sluimer JC, Wang Y, Subramanian M, Brown K, Pattison JS, Robbins J, Martinez J and Tabas I: Macrophage autophagy plays a protective role in advanced atherosclerosis. Cell Metab 15: 545-553, 2012.

49. Dikic I, Johansen T and Kirkin V: Selective autophagy in cancer development and therapy. Cancer Res 70: 3431-3434, 2010.

50. Kim JJ, Lee HM, Shin DM, Kim W, Yuk JM, Jin HS, Lee SH, Cha GH, Kim JM, Lee ZW, et al: Host cell autophagy activated by antibiotics is required for their effective antimycobacterial drug action. Cell Host Microbe 11: 457-468, 2012.

51. White E and DiPaola RS: The double-edged sword of autophagy modulation in cancer. Clin Cancer Res 15: 5308-5316, 2009

52. Pu Z, Wu L, Guo Y, Li G, Xiang M, Liu L, Zhan H, Zhou X and Tan H: LncRNA MEG3 contributes to adenosine-induced cytotoxicity in hepatoma HepG2 cells by downregulated ILF3 and autophagy inhibition via regulation PI3K-AKT-mTOR and beclin-1 signaling pathway. J Cell Biochem 120: 18172-18185, 2019.

53. Li X, Lou X, Xu S, Wang Q, Shen M and Miao J: Knockdown of miR-372 Inhibits nerve cell apoptosis induced by spinal cord ischemia/reperfusion injury via enhancing autophagy by Up-regulating Beclin-1. J Mol Neurosci 66: 437-444, 2018.

54. Cai M, Hu Z, Liu J, Gao J, Liu C, Liu D, Tan M, Zhang D and Lin B: Beclin 1 expression in ovarian tissues and its effects on ovarian cancer prognosis. Int J Mol Sci 15: 5292-5303, 2014.

55. Hennessy BT, Smith DL, Ram PT, Lu Y and Mills GB: Exploiting the PI3K/AKT pathway for cancer drug discovery. Nat Rev Drug Discov 4: 988-1004, 2005

56. Fresno Vara JA, Casado E, de Castro J, Cejas P, Belda-Iniesta C and Gonzalez-Baron M: PI3K/Akt signalling pathway and cancer. Cancer Treat Rev 30: 193-204, 2004.

57. Carnero A, Blanco-Aparicio C, Renner O, Link W and Leal JF: The PTEN/PI3K/AKT signalling pathway in cancer, therapeutic implications. Curr Cancer Drug Targets 8: 187-198, 2008.

58. Arico S, Petiot A, Bauvy C, Dubbelhuis PF, Meijer AJ, Codogno $\mathrm{P}$ and Ogier-Denis E: The tumor suppressor PTEN positively regulates macroautophagy by inhibiting the phosphatidylinositol 3-kinase/protein kinase B pathway. J Biol Chem 276: 35243-35246, 2001.

59. Li H,Zeng J and Shen K: PI3K/AKT/mTOR signaling pathway as a therapeutic target for ovarian cancer. Arch Gynecol Obstet 290 1067-1078, 2014

60. Hu L, Zaloudek C, Mills GB, Gray J and Jaffe RB: In vivo and in vitro ovarian carcinoma growth inhibition by a phosphatidylinositol 3-kinase inhibitor (LY294002). Clin Cancer Res 6 : 880-886, 2000

61. Engel JB, Schönhals T, Häusler S, Krockenberger M, Schmidt M, Horn E, Köster F, Dietl J, Wischhusen J and Honig A: Induction of programmed cell death by inhibition of AKT with the alkylphosphocholine perifosine in in vitro models of platinum sensitive and resistant ovarian cancers. Arch Gynecol Obstet 283: 603-610, 2011.

62. Sun H, Yu T and Li J: Co-administration of perifosine with paclitaxel synergistically induces apoptosis in ovarian cancer cells More than just AKT inhibition. Cancer Lett 310: 118-128, 2011.

63. Yu Y, Hall T, Eathiraj S, Wick MJ, Schwartz B and Abbadessa G: In-vitro and in-vivo combined effect of ARQ 092, an AKT inhibitor, with ARQ 087, a FGFR inhibitor. Anticancer Drugs 28: 503-513, 2017. 
64. Ichikawa K, Abe T, Nagase H, Saito H, Fujita R, Okada M, Yonekura K, Shimomura T and Utsugi T: Abstract C177: TAS-117, a highly selective non-ATP competitive inhibitor of AKT demonstrated antitumor activity in combination with chemotherapeutic agents and molecular targeted drugs. Mol Cancer Ther 12 (Suppl 11): C177, 2013.

65. Son Y, An Y, Jung J, Shin S, Park I, Gwak J, Ju BG, Chung YH, $\mathrm{Na} \mathrm{M}$ and $\mathrm{Oh} \mathrm{S}$ : Protopine isolated from Nandina domestica induces apoptosis and autophagy in colon cancer cells by stabilizing p53. Phytother Res 33: 1689-1696, 2019.

66. Brooks CL and Gu W: Ubiquitination, phosphorylation and acetylation: The molecular basis for $\mathrm{p} 53$ regulation. Curr Opin Cell Biol 15: 164-171, 2003.

67. Brooks CL and Gu W: The impact of acetylation and deacetylation on the p53 pathway. Protein Cell 2: 456-462, 2011

68. Feng Z, Hu W, de Stanchina E, Teresky AK, Jin S, Lowe S and Levine AJ: The regulation of AMPK beta1, TSC2, and PTEN expression by p53: Stress, cell and tissue specificity, and the role of these gene products in modulating the IGF-1-AKT-mTOR pathways. Cancer Res 67: 3043-3053, 2007.

69. Crighton D, Wilkinson S, O'Prey J, Syed N, Smith P, Harrison PR, Gasco M, Garrone O, Crook T and Ryan KM: DRAM, a p53-induced modulator of autophagy, is critical for apoptosis. Cell 126: 121-134, 2006.

70. TasdemirE,Maiuri MC,GalluzziL,Vitale I,Djavaheri-Mergny M, D'Amelio M, Criollo A, Morselli E, Zhu C, Harper F, et al: Regulation of autophagy by cytoplasmic p53. Nat Cell Biol 10: 676-687, 2008

71. Chen $\mathrm{N}$ and Karantza-Wadsworth V: Role and regulation of autophagy in cancer. Biochim Biophys Acta 1793: 1516-1523, 2009.

72. Liang $M$ and Zhao J: Protein expressions of AIB1, p53 and $\mathrm{Bcl}-2$ in epithelial ovarian cancer and their correlations with the clinical pathological features and prognosis. Eur Rev Med Pharmacol Sci 22: 5134-5139, 2018.

73. Chen YN, Ren CC, Yang L, Nai MM, Xu YM, Zhang F and Liu Y: MicroRNA let7d5p rescues ovarian cancer cell apoptosis and restores chemosensitivity by regulating the p53 signaling pathway via HMGA1. Int J Oncol 54: 1771-1784, 2019.

74. Cho CS, Lombard DB and Lee JH: SIRT3 as a regulator of hepatic autophagy. Hepatology 66: 700-702, 2017.

75. Gao J, Feng Z, Wang X, Zeng M, Liu J, Han S, Xu J, Chen L, Cao K, Long J, et al: SIRT3/SOD2 maintains osteoblast differentiation and bone formation by regulating mitochondrial stress. Cell Death Differ 25: 229-240, 2018.

76. Liu H, Li S, Liu X, Chen Y and Deng H: SIRT3 overexpression inhibits growth of kidney tumor cells and enhances mitochondrial biogenesis. J Proteome Res 17: 3143-3152, 2018.

77. Shi H, Deng HX, Gius D, Schumacker PT, Surmeier DJ and Ma YC: Sirt3 protects dopaminergic neurons from mitochondrial oxidative stress. Hum Mol Genet 26: 1915-1926, 2017.

78. Duran A, Amanchy R, Linares JF, Joshi J, Abu-Baker S, Porollo A, Hansen M, Moscat J and Diaz-Meco MT: p62 is a key regulator of nutrient sensing in the mTORC1 pathway. Mol Cell 44: 134-146, 2011.

79. Wang ZG, Li H, Huang Y, Li R, Wang XF, Yu LX, Guang XQ, Li L, Zhang HY, Zhao YZ, et al: Nerve growth factor-induced $\mathrm{Akt} / \mathrm{mTOR}$ activation protects the ischemic heart via restoring autophagic flux and attenuating ubiquitinated protein accumulation. Oncotarget 8: 5400-5413, 2017.

80. Ma Q, Zhang Z, Shim JK, Venkatraman TN, Lascola CD, Quinones QJ, Mathew JP, Terrando N and Podgoreanu MV: Annexin A1 bioactive peptide promotes resolution of neuroinflammation in a rat model of exsanguinating cardiac arrest treated by emergency preservation and resuscitation. Front Neurosci 13: 608, 2019.

81. Tong W, Ju L, Qiu M, Xie Q, Chen Y, Shen W, Sun W, Wang W and Tian J: Liraglutide ameliorates non-alcoholic fatty liver disease by enhancing mitochondrial architecture and promoting autophagy through the SIRT1/SIRT3-FOXO3a pathway. Hepatol Res 46: 933-943, 2016.

82. Zhang M, Lin J, Wang S, Cheng Z, Hu J, Wang T, Man W, Yin T, Guo W, Gao E, et al: Melatonin protects against diabetic cardiomyopathy through Mst1/Sirt3 signaling. J Pineal Res: May 8, 2017 (Epub ahead of print). doi: 10.1111/jpi.12418.

83. Xiang X, Huang J, Song S, Wang Y, Zeng Y, Wu S and Ruan Y: $17 \beta$-estradiol inhibits $\mathrm{H}_{2} \mathrm{O}_{2}$-induced senescence in HUVEC cells through upregulating SIRT3 expression and promoting autophagy. Biogerontology: Mar 14, 2020 (Epub ahead of print). doi: $10.1007 / \mathrm{s} 10522-020-09868-w$.
84.Zhang Y, Kwok-Shing Ng P, Kucherlapati M, Chen F, Liu Y, Tsang YH, de Velasco G, Jeong KJ, Akbani R, Hadjipanayis A, et al: A pan-cancer proteogenomic atlas of PI3K/AKT/mTOR pathway alterations. Cancer Cell 31: 820-832. e3, 2017.

85. Sun Y, Huang YH, Huang FY, Mei WL, Liu Q, Wang CC, Lin YY, Huang C, Li YN,Dai HF and Tan GH: 3'-epi-12 $\beta$-hydroxyfroside, a new cardenolide, induces cytoprotective autophagy via blocking the Hsp90/Akt/mTOR axis in lung cancer cells. Theranostics 8: 2044-2060, 2018.

86. Yang Q, Du WW, Wu N, Yang W, Awan FM, Fang L, Ma J, Li X, Zeng Y, Yang Z, et al: A circular RNA promotes tumorigenesis by inducing c-myc nuclear translocation. Cell Death Differ 24: 1609-1620, 2017.

87. Quan Y, Wang N, Chen Q, Xu J, Cheng W, Di M, Xia W and Gao WQ: SIRT3 inhibits prostate cancer by destabilizing oncoprotein c-MYC through regulation of the PI3K/Akt pathway. Oncotarget 6: 26494-26507, 2015.

88. Wang G, Wang JJ, Wang YZ, Feng S, Jing G and Fu XL: Myricetin nanoliposomes induced SIRT3-mediated glycolytic metabolism leading to glioblastoma cell death. Artif Cells Nanomed Biotechnol 46 (Suppl 3): S180-S191, 2018.

89. Diotte NM, Xiong Y, Gao J, Chua BH and Ho YS: Attenuation of doxorubicin-induced cardiac injury by mitochondrial glutaredoxin 2. Biochim Biophys Acta 1793: 427-438, 2009.

90.Zhao M and Klionsky DJ: AMPK-dependent phosphorylation of ULK1 induces autophagy. Cell Metab 13: 119-120, 2011.

91. Garcia D and Shaw RJ: AMPK: Mechanisms of cellular energy sensing and restoration of metabolic balance. Mol Cell 66: 789-800, 2017.

92. Inoki K, Kim J and Guan KL: AMPK and mTOR in cellular energy homeostasis and drug targets. Annu Rev Pharmacol Toxicol 52: 381-400, 2012

93. Kim J, Kundu M, Viollet B and Guan KL: AMPK and mTOR regulate autophagy through direct phosphorylation of Ulk1. Nat Cell Biol 13: 132-141, 2011.

94. Zhao W,Zhang L, Chen R, Lu H, Sui M, Zhu Y and Zeng L: SIRT3 protects against acute kidney injury via AMPK/mTOR-regulated autophagy. Front Physiol 9: 1526, 2018.

95. Inoki K, Zhu T and Guan KL: TSC2 mediates cellular energy response to control cell growth and survival. Cell 115: 577-590, 2003.

96. Gwinn DM, Shackelford DB, Egan DF, Mihaylova MM, Mery A Vasquez DS, Turk BE and Shaw RJ: AMPK phosphorylation of raptor mediates a metabolic checkpoint. Mol Cell 30: 214-226, 2008.

97. Shackelford DB and Shaw RJ: The LKB1-AMPK pathway: Metabolism and growth control in tumour suppression. Nat Rev Cancer 9: 563-575, 2009.

98. Zhang M, Deng YN, Zhang JY, Liu J, Li YB, Su H and Qu QM: SIRT3 protects rotenone-induced injury in SH-SY5Y cells by promoting autophagy through the LKB1-AMPK-mTOR pathway. Aging Dis 9: 273-286, 2018.

99. Bazhin AV, Philippov PP and Karakhanova S: Reactive oxygen species in cancer biology and anticancer therapy. Oxid Med Cell Longev 2016: 4197815, 2016.

100.Shi L, Zhang T, Zhou Y, Zeng X, Ran L, Zhang Q, Zhu J and Mi M: Dihydromyricetin improves skeletal muscle insulin sensitivity by inducing autophagy via the AMPK-PGC-1alpha-Sirt3 signaling pathway. Endocrine 50: 378-389, 2015

101. Lv W, Sui L, Yan X, Xie H, Jiang L, Geng C, Li Q, Yao X, Kong Y and Cao J: ROS-dependent Atg4 upregulation mediated autophagy plays an important role in Cd-induced proliferation and invasion in A549 cells. Chem Biol Interact 279: 136-144, 2018.

102. Li ZY, Yang Y, Ming M and Liu B: Mitochondrial ROS generation for regulation of autophagic pathways in cancer. Biochem Biophys Res Commun 414: 5-8, 2011.

103. Xia C, He Z, Liang S, Chen R, Xu W, Yang J, Xiao G and Jiang S: Metformin combined with nelfinavir induces SIRT3/mROS-dependent autophagy in human cervical cancer cells and xenograft in nude mice. Eur J Pharmacol 848: 62-69, 2019.

104. Qiu X, Brown K, Hirschey MD, Verdin E and Chen D: Calorie restriction reduces oxidative stress by SIRT3-mediated SOD2 activation. Cell Metab 12: 662-667, 2010.

105. Tan WX, Xu TM, Zhou ZL, Lv XJ, Liu J, Zhang WJ and Cui MH: TRP14 promotes resistance to cisplatin by inducing autophagy in ovarian cancer. Oncol Rep 42: 1343-1354, 2019. 
106. Qiu S, Sun L, Zhang Y and Han S: Downregulation of BAG3 attenuates cisplatin resistance by inhibiting autophagy in human epithelial ovarian cancer cells. Oncol Lett 18: 1969-1978, 2019.

107. Xie Z, Guo Z, Wang Y, Lei J and Yu J: Protocatechuic acid inhibits the growth of ovarian cancer cells by inducing apoptosis and autophagy. Phytother Res 32: 2256-2263, 2018.

108. Zhu H, Diao S, Lim V, Hu L and Hu J: FAM83D inhibits autophagy and promotes proliferation and invasion of ovarian cancer cells via PI3K/AKT/mTOR pathway. Acta Biochim Biophys Sin (Shanghai) 51: 509-516, 2019.

109. Wu Y, Gao WN, Xue YN, Zhang LC, Zhang JJ, Lu SY, Yan XY, Yu HM, Su J and Sun LK: SIRT3 aggravates metformin-induced energy stress and apoptosis in ovarian cancer cells. Exp Cell Res 367: 137-149, 2018

110. Zou G, Bai J, Li D and Chen Y: Effect of metformin on the proliferation, apoptosis, invasion and autophagy of ovarian cancer cells. Exp Ther Med 18: 2086-2094, 2019.

111. Li X, Su J, Xia M, Li H, Xu Y, Ma C, Ma L, Kang J, Yu H, Zhang Z and Sun L: Caspase-mediated cleavage of Beclin1 inhibits autophagy and promotes apoptosis induced by S1 in human ovarian cancer SKOV3 cells. Apoptosis 21: 225-238, 2016.
112. Liu N, Xu Y, Sun JT, Su J, Xiang XY, Yi HW, Zhang ZC and Sun LK: The BH3 mimetic S1 induces endoplasmic reticulum stress-associated apoptosis in cisplatin-resistant human ovarian cancer cells although it activates autophagy. Oncol Rep 30: 2677-2684, 2013.

113. Yang X, Xiang X, Xia M, Su J, Wu Y, Shen L, Xu Y and Sun L: Inhibition of JNK3 promotes apoptosis induced by BH3 mimetic S1 in chemoresistant human ovarian cancer cells. Anat Rec (Hoboken) 298: 386-395, 2015

114. Yang Y, Li N, Chen T, Zhang C, Li J, Liu L, Qi Y, Zheng X, Zhang $\mathrm{C}$ and Bu P: Sirt3 promotes sensitivity to sunitinib-induced cardiotoxicity via inhibition of GTSP1/JNK/autophagy pathway in vivo and in vitro. Arch Toxicol 93: 3249-3260, 2019.

c) (7) $\odot$ This work is licensed under a Creative Commons Attribution-NonCommercial-NoDerivatives 4.0 International (CC BY-NC-ND 4.0) License. 